\title{
A new methodologic approach for clinico-pathologic correlations in invasive placenta previa accreta
}

Eric Jauniaux, MD, PhD; Ahmed M Hussein, MD; Nurit Zosmer, MD; Rana M Elbarmelgy; MsC; Rasha A Elbarmelgy; MsC; Hizbullah Shaikh, MD; Graham J Burton. MD, DSc

From the EGA Institute for Women's Health, Faculty of Population Health Sciences, University College London (UCL), London, UK (Dr Jauniaux); the Department of Obstetrics and Gynecology, University of Cairo, Cairo, Egypt (Drs Hussein, RM Elbarmelgy and RA Elbarmelgy; The Fetal Medicine Research Institute, Harris Birthright Research Centre (Dr Zosmer) and Department of Histopathology (Dr Shaikh), Kings College Hospital, London, UK; The Centre for Trophoblast Research, Department of Physiology, Development and Neuroscience, University of Cambridge, Cambridge, UK (Dr Burton).

Corresponding author: Eric Jauniaux, $M D, P h D, F R C O G$ Institute for Women's Health, University College London, 86-96 Chenies Mews, London WC1E 6HX, UK. e.jauniaux@ucl.ac.uk

No funding was obtained for this project.

The authors have no conflict of interest to declare.

Word count: 4252

Short title: Clinico-pathologic correlation for invasive placenta previa accreta.

\section{Condensation}

Intra-operative and immediate post-operative gross examination provides accurate data on uterine dehiscence, vascular changes and depth of villous invasion in placenta previa accreta. 


\section{AJOG at a Glance}

- A. Why was this study conducted? To evaluate the value of intra-operative and immediate post-operative gross examination of hysterectomy specimens with placenta in-situ compared to standard pathologic examination.

- B. What are the key findings? The intra-operative and immediate postoperative examination is more efficient and cost-effective than standard histopathology examination with shorter reporting time and provides accurate stratification of the depth and lateral extension of villous invasiveness which are essential to confirm the clinical diagnosis in placenta accreta spectrum.

- C. What does this study add to what is already known? Our proposed methodology provides additional data to standard pathological examination, in particular regarding the anatomical changes to the vasculature and myometrium of the lower uterine segment, and engages more efficiently perinatal pathologists into a multidisciplinary team. 


\section{ABSTRACT}

BACKGROUND: The development of new management strategies for women presenting with placenta accreta spectrum requires quality epidemiology data which have so far been limited by the high variability in clinical and histopathologic data confirming the diagnosis at birth.

OBJECTIVE: To evaluate the role of a new methodologic approach for the correlation of clinical and pathological data for women with a history of prior cesarean delivery diagnosed prenatally with placenta previa accreta.

STUDY DESIGN: A modified pathologic technique for gross examination of hysterectomy specimens with placenta in-situ consisting of intra-operative examination, immediate post-operative examination and guided histologic sampling was used prospectively in a cohort of 24 patients with singleton pregnancies complicated by placenta low-lying/placenta previa accreta. The maternal characteristics, detailed ultrasound findings, surgical outcomes and histopathologic examination were compared with those of a group of 24 patients with similar clinical characteristics where a standard pathologic examination method was used.

RESULTS: The median reporting time for obtaining the complete histopathology results including the microscopic examination was significantly shorter (7 vs 15 days; $\mathrm{P}<0.001)$ and the median number of samples taken for histologic examination significantly lower (4 vs 14 samples; $\mathrm{P}<0.001$ ) in the study group than in the controls. The number of histologic slides showing villous invasion was significantly higher (2 vs 1 slides; $\mathrm{P}=0.002$ ) and the ratio of the number of samples taken to the numbers of slides confirming villous invasion was significantly lower (2 vs $9 ; \mathrm{P}<0.001)$ in the study group than in the controls. In all cases of the study group, intra-operative 
examination identified dense tangled bed of vessels or multiple vessels running laterally and cranio-caudally in the uterine serosa above the placental insertion which were no longer visible at the immediate gross post-operative examination of the hysterectomy specimens and anterior wall dehiscence in 20 cases. Immediate postoperative dissection enables the differential diagnosis between focal and large increta areas and between abnormally adherent placenta and invasive placenta accreta.

CONCLUSIONS: Valuable clinical information on the serosal vascularity, uterine dehiscence and extension of the accreta area is added with the description of the macroscopic examination during the surgical procedure and immediate dissection of the specimen. This methodological approach is cost-effective and increases the quality of the histologic sampling and thus provides accurate correlations with the clinical data and more accurate epidemiologic data collection. Perinatal pathologists should be part of multidisciplinary teams involved the management placenta accreta spectrum disorders.

Key words: Placenta previa accreta, increta, percreta, histopathology, grading. 


\section{Introduction}

The main factor associated with the development of both placenta previa and placenta accreta spectrum disorders is a prior caesarean delivery, and the risks of abnormal placentation in subsequent pregnancies increases with the number of previous caesarean sections. ${ }^{1-6}$ The scar tissue created by a caesarean delivery in the lower uterine segment myometrium stimulates both implantation of the blastocyst in the area of the scar and the abnormal adherence or invasion of placental villi within the scar tissue. ${ }^{7,8}$ Thus placenta previa and placenta accreta spectrum are often combined, and women with a prior history of caesarean section, presenting with a low-lying placenta or placenta previa, are now the group of pregnant women with the highest risk of placenta accreta spectrum disorders. ${ }^{9}$ The combination of both conditions leads to high maternal morbidity and mortality due to massive obstetric hemorrhage at delivery. ${ }^{6,10}$ Placenta accreta spectrum has been reported in primiparous after operative hysteroscopy, myomectomy, suction curettage, surgical termination and endometrial ablation, but the corresponding incidences are low. ${ }^{11}$. Very rare cases of placenta accreta spectrum have also been described in women with bicornuate uterus, adenomyosis, submucous fibroids and myotonic dystrophy. ${ }^{6}$

Before the advent of high resolution ultrasonography at the end of the 1980s, the diagnosis of placenta accreta spectrum was almost exclusively clinical using criteria initially reported in 1937 by Irving and Hertig based on a cohort series of 20 cases. ${ }^{12}$ They described these cases as presenting at birth with abnormal adherence of the placenta to the underlying uterine wall, either in whole or in part, due to the absence of the decidual/Nitabuch layer between the tip of anchoring villi and superficial myometrium. These criteria were further expanded by subsequent authors 
to include piecemeal removal of the placenta, no evidence of placental separation after 20 min despite active management using utero-tonics, and/or bleeding from the placental site in a well-contracted uterus during caesarean delivery ${ }^{13,14}$. Overall, these clinical symptoms are very similar to those of non-accreta placental retention whereas the absence of decidua is an elusive histological criterion as such areas are found with increasing incidence with advancing gestation in pregnancies without evidence of placenta accreta spectrum. ${ }^{8}$

Lukes et $\mathrm{al}^{15}$ were the first in 1966 to propose a histological classification for placenta accreta based on the depth of the villous penetration of the myometrium and an inclusive terminology, i.e. placenta accreta spectrum. They also highlighted the fact that villous penetration of the myometrium is rarely uniform, and that both adherent and invasive villi may co-exist in the same specimen. Although, Irving and Hertig did not have cases of invasive placenta accreta in their series ${ }^{12}$, many authors of modern cohort studies continue to use their clinical criteria for both adherent and invasive placenta accreta, often without detailed histopathologic confirmation. Only 34 out of 1078 cases, reported in cohort studies on the prenatal diagnosis of placenta accreta spectrum between the first prenatal ultrasound description of the condition by Tabsh et $\mathrm{al}^{16}$ in 1982 and 2016, provided detailed correlations between ultrasound findings and the grade of placental tissue invasiveness. ${ }^{17}$ This can explain the wide heterogeneity in the evaluation of the prevalence of placenta accreta spectrum and its different grades in the general obstetric population. ${ }^{18}$

The risk of life-threatening complications is particularly high with the invasive grades due to involvement of the main branches of uterine arteries and the possible invasion of the bladder wall and surrounding pelvic vessels..$^{8,19,20}$. Thus, confirmation of the depth of villous invasion of the uterine myometrium in cases of placenta 
accreta spectrum is essential to improve prenatal detection, outcome and management protocols. Dannheim et $\mathrm{al}^{21}$ have recently reviewed their protocol for gross dissection and microscopic examination of surgical specimens in cases of placenta accreta spectrum suspected prenatally, and found that confirmation of the diagnosis can be very difficult if the surgeon has attempted to remove the placenta, or impossible in cases of conservative management where the whole placenta is left in situ. The aim of this study was therefore to develop a practical method to overcome the obstacles of standard histopathologic examination and enhance the accuracy of clinic-pathologic correlations in patients with a history of cesarean delivery presenting with a low-lying/ placenta previa and prenatal ultrasound signs of placenta accreta spectrum.

\section{Materials and Methods}

We conducted a prospective cohort study of 24 consecutive patients with singleton pregnancies diagnosed prenatally with low-lying/placenta previa accreta. All patients were referred for delivery in the third trimester by an expert multidisciplinary team at the University of Cairo between September 2018 and June 2019. In all cases, a detailed transabdominal and transvaginal ultrasound examination of the placenta, uterus and pelvis was performed within 24 hours before surgery (GE Voluson ${ }^{\circledR}$ E10, GE Medical System, Zipf, Austria).

All patients were managed by elective caesarean hysterectomy and photographs of the uterus were obtained intra-operatively before delivery of the fetus. The corresponding images were analysed for the presence and size (focal or large) of anterior wall uterine dehiscence (Figure 1), placental tissue invasion through the uterine wall and abnormally increased vascularity of the uterine serosa 
over the placental bed i.e. dense tangled bed of vessels or multiple vessels running in the anterior uterine serosa or perimetrium as previously described (Figures 1 and 2)..$^{8,22,23}$ In all cases, immediately after the surgical procedure, the hysterectomy specimen was orientated anteriorly, measured and photographed and cut in slices of 2-3 cm thick. Areas of abnormal placental attachment that could not be separated by gentle digital dissection were identified and documented (Figures 3 and 4). The surface of these areas was evaluated for size (focal or large) and sampled for microscopic examination to include the full thickness of the uterine wall and at least half of the thickness of the corresponding placental tissue. All samples were immediately fixed in $10 \%$ neutral buffered formalin and processed for histologic examination and stained with hematoxylin and eosin (H\&E). All cases were reviewed by the multidisciplinary team including the surgical team and perinatal pathologists.

For comparison, we retrospectively reviewed the ultrasound and histopathologic data of 24 patients with singleton pregnancies diagnosed with lowlying/placenta previa accreta and referred for delivery in the third trimester by an expert multidisciplinary team at the King's College Hospital between January 2015 and January 2019. Only cases managed by caesarean hysterectomy and for which maternal characteristics, detailed ultrasound findings, surgical outcomes and histopathologic examination were available, were included in the control group. All ultrasound examinations in this group were performed on GE Voluson ${ }^{\circledR} 730$ (GE Medical System, Zipf, Austria). The hysterectomy specimens are sent to the pathology department for gross examination in the fixed state and dissection by the duty pathologist as per the Royal College of Pathologists guidelines for the tissue pathways for gynaecological pathology (https://www.rcpath.org). As per local protocol for the gross examination of uterine specimens, the uterus is then opened laterally 
and replaced in 10\% neutral buffered formalin. After fixing for a minimum of 6-7 days in, samples are taken from areas suspected of abnormal adherence or invasion, processed and stained with H\&E for histologic examination.

In all cases of both groups, the fetal ultrasound measurements and diagnosis of abnormal placentation were obtained prenatally by expert maternal-fetal medicine physicians. The placenta was recorded as "low lying" when the edge was $0.5-2 \mathrm{~cm}$ from the internal os of the uterine cervix. When the placenta was $<0.5 \mathrm{~cm}$ from the internal os or completely covering it, it was defined as placenta previa (marginal or complete). The prenatal ultrasound diagnosis of placenta accreta spectrum disorder was made by maternal-fetal medicine physicians experienced with the condition using the standardised reporting pro-forma proposed by the AIP international expert group. ${ }^{24}$ For the score of placental lacunae, we used the classification proposed by Finberg and Williams $(0=$ none; $1+=1-3 ; 2+=4-6 ; 3+=>6) .{ }^{25}$

All patients were managed according to their local unit's protocol. Institutional ethical committee approval was obtained prior to the start of this study. We were advised that additional patient consent was not required as all ultrasound records were examined within the centre where it was undertaken, basic clinical data were collected using a standard clinical audit protocol, hysterectomy specimens were systematically sent for detailed histopathologic examination and all data were fully anonymised before analysis.

\section{Statistical analysis}

StatGraphic-plus Version 3 data analysis and statistical software package (Manugistics, Rockville, MD) was used to analyse the data. A standard Kurtosis analysis indicated that some values were not normally distributed and the data are therefore presented as median and interquartile range (IQR). Categorical variables 
were compared between groups using the Pearson's chi-square test or Fisher's exact test when samples sizes were small. Continuous variables were compared using a Mann-Whitney (Wilcoxon) W rank test at the 95\% confidence interval $(\mathrm{CI}) . \mathrm{A}$ $P$ value $<0.05$ was considered significant.

\section{Results}

The maternal demographics and pathology technical characteristics of the study and control groups are displayed and compared in Table 1. The median maternal age was significantly $(p=0.004)$ lower and the median number of prior caesarean sections significantly $(p=0.005)$ higher in the study group than in the controls. There was no significant difference between the groups for gravidity and parity. The median reporting time for obtaining the complete histopathology results including the histologic examination was significantly $(\mathrm{P}<0.001)$ shorter and the median number of samples taken for microscopic examination significantly $(\mathrm{P}<0.001)$ lower in the study group than in the controls. The number of histologic slides showing villous invasiveness was significantly $(P=0.002)$ higher and the ratio of the number of samples taken to the numbers of slides confirming villous invasiveness was significantly $(P<0.001)$ lower in the study groups than in the controls. There was no significant difference in the distribution of adherent versus invasive cases between the groups.

Table 2 shows the main ultrasound features and peri-operative macroscopic findings in the study group. There was only one low-lying placenta and one marginal placenta previa. All the other cases were partially or completely covering the inner os of the uterine cervix. A thin myometrium ( $<1 \mathrm{~mm}$ in thickness) was found in 19 (79.2\%) cases. Fourteen (58.3\%) cases presented with six or more placental 
lacunae (Grade 3+) and lacunar feeder vessels were found in $13(54.2 \%)$ of these cases. Feeder vessels were also found in a case which presented with 4-6 lacunae (score 2+). Areas with loss of clear zone were found in all cases and hypervascularity of the subplacental zone was recorded in $22(91.7 \%)$ cases. There was a similar distribution for the following ultrasound features in the controls; thin myometrium (21 cases); loss of clear zone (24 cases) and hypervascularity of uterovesical interface/subplacental zone (22 cases). The number of cases presenting with six or more placental lacunae (6 control cases) and feeder vessels (4 control cases) was significantly (Chi-square with Yates correction; $\mathrm{P}=0.041$ and $\mathrm{P}=0.016$; respectively) higher in the control than in the study group.

A dense tangled bed of vessels or multiple vessels running laterally and cranio-caudally in the uterine serosa superficial to the placental insertion (Figure 2) were visible macroscopically during the intra-operative examination in all 24 cases of the study group. These features were no longer visible at the immediate gross postoperative examination of the hysterectomy specimens (Figures 3 and 4). Intraoperative examination of the 22 invasive cases from the study group (Table 3) also found anterior wall dehiscence in 20 , of which nine were classified as focal and 11 as large (Figure 1).

The immediate post-operative examination, after dissection of the hysterectomy specimen, showed focal invasive area (Figure 3) in nine (37.5\%) cases and large invasive areas (Figure 4) in 13 (54.2\%) cases. In seven cases presenting with a large area of invasive tissue, and in one case with focal invasive villous tissue and, the gross examination also showed dilated vessels protruding from the uterine wall into the uterine cavity, away from the invasive areas (Figures $3 \& 6$ ). The 
corresponding lacunae grading was $3+$ in five cases with focal invasive areas and in nine cases with large invasive areas. A similar distribution was found for feeder vessels between cases presenting with focal and large dehiscence, and between cases presenting with focal and large invasive areas.

In two cases of the study group, the placenta did not spontaneously separate during the caesarean section after delivery of the fetus. In those cases, the placenta could be entirely detached digitally from the uterine wall of the corresponding hysterectomy specimen during the immediate post-operative examination (Figure 5). In those cases, microscopically, there were areas where the villi were directly attached to the myometrium but no evidence of villous invasion. The other 22 cases showed evidence of invasive villous tissue at the time of immediate post-operative dissection, and were confirmed by histology as increta. Eight cases in the control group were classified perinatally as percreta. In four of these cases, invasive villous

tissue was found within the myometrium on histology but not in the serosa, indicating the diagnosis of placenta increta. In the others, the microscopic examination showed areas of direct apposition of the villous tissue suggesting an abnormally adherent placenta. In none of the cases in both groups was there evidence on histology of villous invasion within nor beyond the uterine serosa.

\section{Comment}

\section{Principal findings of the study}

Our data add to prior classical studies of the histopathology of placenta accreta spectrum and show that intra-operative macroscopic description of the gross uterine features on opening of the abdomen combined with immediate post-operative 
dissection of the hysterectomy specimen improves the accuracy of the clinical diagnosis, and in particular the differential diagnosis between the different grades of accreta placentation. This methodological approach is also more efficient and costeffective than standard histopathology examination, with shorter reporting time for complete histopathology results including microscopic examination and lower number of samples needed to confirm the grade of villous invasiveness.

\section{Comparison with existing literature}

Only a few pathology studies have tried to explore the histopathology in placenta accreta spectrum disorders, and only one ${ }^{19}$ describes the macroscopic features of hysterectomy specimens with placenta in-situ. By contrast, there are many series on the prenatal diagnosis of placenta accreta spectrum with ultrasound and/or magnetic resonance imaging, but few that provide detailed clinical and histopathology data on the grade of villous invasiveness. ${ }^{17}$ In a recent systematic review and meta-analysis of 29 population studies, we observed large amounts of heterogeneity between studies for all clinical and pathologic parameters investigated. ${ }^{18}$ The overall prevalence of placenta accreta spectrum at delivery was found to range between 1 in 1000 and 1 in 40,000 births. In the ten studies that provide histopathologic data, the distribution of the different grades varied widely i.e. between 35 and $82 \%$ for placenta creta (adherenta), 4 and $44 \%$ for placenta increta and 7 and $34 \%$ for placenta percreta. ${ }^{18}$ These findings highlight methodological inconsistencies between studies with regards to clinical criteria used for the diagnosis of the condition at birth and for the histopathologic confirmation of the diagnosis and in particular for differential diagnosis between adherent and invasive accreta placentation. 
In all of the 20 cases included in Irving and Hertig's original series of placenta accreta, the diagnosis was based on the failure of the placenta to deliver spontaneously and/or the difficulty of removing it at birth, and the absence of decidua between the anchoring villi and the superficial myometrium on microscopic examination. ${ }^{12}$ The pathology reports of their 15 cases that required a hysterectomy only describe the placenta as being firmly attached to the uterine wall in eight cases. They described the lateral extension of an abnormally adherent area in only one case (case 13) where around one-third of the placenta was embedded within the cervix and could not be digitally separated from the uterus. In their series of 21 cases, Luke et $\mathrm{al}^{15}$, found that most hysterectomy specimens arrive to the laboratory so distorted by attempts by the surgeon to remove the placenta that it is impossible to judge accurately the surface of the accreta areas. This methodological issue has been raised in other pathology studies. ${ }^{21,29}$

Increased vascularity of the placental bed is one of the main prenatal ultrasound diagnostic signs of placenta accreta spectrum..$^{17,26-28}$ Subplacental hypervascularity has been reported in over $85 \%$ of the cases published in the international literature between 1982 and $2016^{17}$, and is due to the vascular remodelling of the deep uterine vessels beyond the level of the spiral arteries. ${ }^{8}$ This leads to the excessive dilatation of the utero-placental circulation ${ }^{28}$ and the development of intraplacental lacunae but also to abnormal enlargement of the vasculature on the outer surface of the uterus above the placental implantation site. The latter vascular changes can be easily identified upon entry to the abdomen (Figures 1 and 2); however, very few authors of prenatal diagnostic cohorts have described the gross intra-operative features associated with accreta placentation. ${ }^{26,27}$ During the immediate post-operative gross examination in our study group, we also 
found dilated vessels protruding from the uterine wall towards the uterine cavity, away from the invasive areas in seven cases (Figure 3). These vessels are probably dilated arterio-venous anastomoses part of extensive vascular anastomotic network present in the subplacental myometrium during normal pregnancy. ${ }^{31}$ Our data suggests that even focal villous invasion could have an impact beyond the invasive area. None of the old ${ }^{12,15,29}$ or more recent ${ }^{21,30}$ pathology studies of hysterectomy specimens with placenta "in situ" reported on macroscopic evidence of an excessive dilatation of the uterine vasculature in the accreta area. This can be explained by the fact that routine pathologic examination takes place usually several hours after surgery or after fixing of the hysterectomy specimen in formalin for several days by which time the changes in uterine vasculature associated with invasive placentation are no longer visible macroscopically.

Thinning of the myometrial zone to less than $1 \mathrm{~mm}$ and the absence of the hypoechoic clear zone between the placenta and posterior bladder wall on ultrasound have been reported in 66 and $98 \%$, respectively, of all the cases of placenta accreta spectrum published in the international literature between 1982 and 2016. ${ }^{17}$ Both features are associated with the highest agreement between observer ${ }^{32}$ and have been used by many authors as strong markers of accreta placentation on ultrasound. The myometrium will become thinner with advancing gestation. ${ }^{8}$ This thinning effect is more pronounced in the third trimester when the lower uterine segment is further stretched by the combined action of the fetal presentation and Braxton-Hicks uterine contractions. When the placenta develops underneath a caesarean scar defect, the myometrium of the lower segment is partially or completely replaced by scar tissue and thus likely to be even thinner, independently of any abnormal villous invasion. All the patients included in our study had a history 
of one or more caesarean sections and presented with a low-lying/placenta previa. This can explain the higher incidence of myometrial thinning found in both the study group and controls (79.2\% versus $87.5 \%$ ). This finding is supported by the presence of focal and large area of dehiscence in one of the two and 20 of the 22 abnormally adherent and invasive cases, respectively. Ultrasound evaluation of the myometrium thickness has so far not been standardised, and myometrium thinning is currently defined as an area of the uterine wall that is less that $1 \mathrm{~mm}$ on ultrasound anywhere under the placental bed. Further studies are needed to evaluate the lateral extension and changes in myometrium thickness with advancing gestation on ultrasound examination correlated with gross examination at birth.

The clear zone is a radiologic term that is supposed to represent the layer of tissue between the villous tissue and the deep myometrium and which includes the decidua and the superficial myometrium containing the utero-placental vessels. ${ }^{8}$ As for myometrium thickness, the decidual layer becomes thinner and discontinuous as pregnancy advances. The superficial myometrium under the placental bed become also more heterogeneous due to the progressive dilatation of the utero-placental circulation. The absence of the clear zone on ultrasound probably represents the abnormal extension of the placental villi through the decidua basalis into the myometrium or the poor development of this layer around scar area. ${ }^{8}$ Like myometrial thinning this sign is not specific of accreta placentation, in particular when the placenta is implanted in a lower uterine segment remodelled by multiple caesarean scars.

\section{Clinical implications}

According to Dannheim et $\mathrm{al}^{21}$, pathologists often impose a dogmatic approach to the diagnosis of placenta accreta, requiring an ideal textbook image of villi sitting on 
top of the myometrium and thus reporting cases with obvious gross invasion without this "requirement" as merely 'suspicious for accreta'. Furthermore, the pre-operative anatomy of the uterus and in particular of the lower segment may be distorted by the surgical procedure making it difficult for the pathologist to orientate the hysterectomy specimen to differentiate between areas of accreta placentation and areas of dehiscence.

The gold standard diagnostic criteria for invasive placentation is the microscopic evidence of villous tissue invading the myometrium. Accurate identification of the invasive area with guided sampling is therefore essential to accurate confirm the clinical diagnosis. The degree of villous adhesion or invasion is rarely uniform across the placental bed and many cases of placenta accreta spectrum have both adherent and invasive areas. ${ }^{15}$ This can make the microscopic diagnosis of accreta placentation difficult, in particular, if the accreta area of the myometrium is not extensively sampled. We found that digital separation of the placental tissue from the uterine wall in fresh hysterectomy specimens allowed us to differentiate between abnormally adherent and invasive areas, to evaluate the area of villous tissue invasion and to accurately obtain a sample for histology to confirm the diagnosis of villous myometrial invasion in all cases. All the cases included in this study were anterior low-lying/ placenta previa accreta but our method could be also applied for invasive placentation in other area of the uterine cavity. By contrast, as shown in our control group, delayed gross examination of hysterectomy specimens does not allow the pathologist to easily identify of these areas and this may be even more difficult after prolonged formalin fixation.

Patients with a history of multiple lower-segment caesarean deliveries will have an anterior myometrial wall largely consisting of fibrotic scar tissue. ${ }^{8}$ Myofibre 
loss and the excessive accumulation of collagen impairs the function of muscular tissue, which loses elasticity and becomes more prone to dehiscence and rupture in subsequent pregnancies. Lower-segment dehiscence becomes more pronounced as pregnancy advances due to the pressure of the fetus and uterine contractions, both of which increase the disruption of the fibrotic tissue. ${ }^{8}$ As shown in our study group, this creates areas of dehiscence in the lower uterine segment covered only with perimetrium also called uterine "windows" (Figures 1 and 2). In these cases, the portion of the placenta that is implanted underneath is often visible through the uterine window independently of any villous tissue invading the serosa and/or the surrounding myometrium (Figure 3). In eight cases in the control group, the placenta was classified as percreta by the operating team at delivery. In four of these cases, there was no evidence invasive villous tissue within or around the scar area. The corresponding surgical and pathology reports did not describe in detail the macroscopic features of the hysterectomy specimens, and thus it is likely that these cases were not cases of placenta percreta. These findings suggest that areas of dehiscence of the lower uterine segment in patients with a history of prior caesarean deliveries presenting with placenta previa can be easily mistaken intra-operatively as placenta percreta and can explain the wide variation in the incidence of this placentation anomaly reported in the literature. ${ }^{18}$ These findings also highlight the importance of a close collaboration between the surgical and pathology team.

\section{Strengths and limitation of the study}

Our study has a number of strengths compared to other contemporary published studies. The study group was evaluated prospectively allowing direct correlations between ultrasound imaging and gross intra-operative and immediate post-operative features. The intra-operative and immediate post-operative gross examination of 
hysterectomy specimens with placenta in-situ and guided sampling for histological examination allowed us to accurately stratify our cases for the depth and extent of villous invasiveness. This methodological approach should also be useful in cases of conservative management, and in particular in cases where partial myomectomy of the accreta area can be achieved.

The weakness of the study rests on the retrospective design of the control group, the difference in maternal characteristics between the group and the relatively small sample size of both groups which does not allow for more detailed correlations between prenatal ultrasound imaging, clinical findings at birth and histopathologic findings. This may have created a bias in favour of the method used to evaluate study group. Further larger prospective studies are now required to evaluate the relationships between specific ultrasound signs and histopathology results and their impact on the accuracy of prenatal diagnosis, management and outcome of the different grades of placenta accreta spectrum.

\section{Conclusions}

Health provision for the development of centres of excellence with a specialist multidisciplinary team, blood bank and intensive care infrastructure to manage patients with placenta accreta spectrum requires an accurate evaluation of its prevalence and outcome which entails the use of standardized perinatal diagnostic protocols. The precise description of the extension of the abnormal placental attachment, degree of villous invasiveness and/or spatial relationship of the accreta villous tissue with the previous caesarean section scar, the cervix, and main uterine circulation are essential to obtain valuable epidemiology data and evaluate the outcome of different management strategies. 
Our proposed methodology is simple and cost-effective in obtaining additional valuable data to standard pathologic examination and engages more efficiently perinatal pathologists into the multidisciplinary team. Closer collaboration between clinicians and pathologists and the use of this protocol facilitates immediate correlation with imaging and intra-operative findings as well as standardized tissue sampling for accurate differential diagnosis between the different grades of villous invasiveness. This approach should also provide valuable data for clinical training, implementation of guidelines and changes in clinical practice behaviour and help us to better understand the pathophysiology of placenta accreta spectrum. 


\section{References}

1. Ananth CV, Smulian JC, Vintzileos AM. The association of placenta previa with history of cesarean delivery and abortion: a meta-analysis. Am J Obstet Gynecol. 1997;177:1071-8.

2. Silver RM, Landon MB, Rouse DJ, Leveno KJ, Spong CY, Thom EA et al.; National Institute of Child Health and Human Development Maternal-Fetal Medicine Units Network. Maternal morbidity associated with multiple repeat cesarean deliveries. Obstet Gynecol 2006;107:1226-32.

3. Marshall NE, Fu R, Guise JM. Impact of multiple cesarean deliveries on maternal morbidity: a systematic review. Am J Obstet Gynecol 2011;205:262.e1-8.

4. Fitzpatrick KE, Sellers S, Spark P, Kurinczuk JJ, Brocklehurst P, Knight M. Incidence and risk factors for placenta accreta/increta/percreta in the UK: a national case-control study. PLoS One. 2012;7:e52893.

5. Thurn L, Lindqvist PG, Jakobsson M, Colmorn LB, Klungsoyr K, Bjarnadóttir $\mathrm{RI}$, et al. Abnormally invasive placenta-prevalence, risk factors and antenatal suspicion: results from a large population-based pregnancy cohort study in the Nordic countries. BJOG 2016;123:1348-1355.

6. Jauniaux E, Chantraine F, Silver RM, Langhoff-Roos J; FIGO Placenta Accreta Diagnosis and Management Expert Consensus Panel. FIGO consensus guidelines on placenta accreta spectrum disorders: Epidemiology. Int J Gynaecol Obstet 2018;140:265-273.

7. Jauniaux E, Burton GJ. Pathophysiology of Placenta Accreta Spectrum Disorders: A Review of Current Findings. Clin Obstet Gynecol. 2018;61:743754. 
8. Jauniaux E, Collins SL, Burton GJ. Placenta accreta spectrum:

Pathophysiology and evidence-based anatomy for prenatal ultrasound imaging. Am J Obstet Gynecol. 2018;218:75-87.

9. Jauniaux E, Bhide A. Prenatal ultrasound diagnosis and outcome of placenta previa accreta after caesarean delivery: a systematic review and metaanalysis. Am J Obstet Gynecol 2017;217:27-36.

10. Silver RM, Branch DW. Placenta Accreta Spectrum. N Engl J Med. 2018;378:1529-36.

11. Baldwin HJ, Patterson JA, Nippita TA, Torvaldsen S, Ibiebele I, Simpson JM, Ford JB. Antecedents of abnormally invasive placenta in primiparous women: risk associated with gynecologic procedures. Obstet Gynecol. 2018;131:22733.

12. Irving C, Hertig AT. A study of placenta accreta. Surgery, Gynecol Obstet 1937;64:178-200.

13. Gielchinsky Y, Rojansky N, Fasouliotis SJ, Ezra Y. Placenta accreta--summary of 10 years: a survey of 310 cases. Placenta. 2002;23:210-4.

14. Weeks AD. The retained placenta. Best Pract Res Clin Obstet Gynaecol. 2008;22:1103-17.

15. Luke RK, Sharpe JW, Greene RR. Placenta accreta: The adherent or invasive placenta. Am J Obstet Gynecol 1966;95:660-8.

16. Tabsh KM, Brinkman CR 3rd, King W. Ultrasound diagnosis of placenta increta. J Clin Ultrasound. 1982;10:288-90.

17. Jauniaux E, Collins SL, Jurkovic D, Burton GJ. Accreta placentation. A systematic review of prenatal ultrasound imaging and grading of villous invasiveness. Am J Obstet Gynecol. 2016; 215:712-21. 
18. Jauniaux E, Bunce C, Grønbeck L, Langhoff-Roos J. Prevalence and main outcomes of placenta accreta spectrum: a systematic review and metaanalysis. Am J Obstet Gynecol 2019;221:208-18.

19. Creanga AA, Bateman BT, Butwick AJ, Raleigh L, Maeda A, Kuklina E, et al. Morbidity associated with cesarean delivery in the United States: is placenta accreta an increasingly important contributor? Am J Obstet Gynecol. 2015;213:384.e1-11.

20. Marcellin L, Delorme P, Bonnet MP, Grange G, Kayem G, Tsatsaris V et al. Placenta percreta is associated with more frequent severe maternal morbidity than placenta accreta. Am J Obstet Gynecol. 2018;219:193.e1-193.e9.

21. Dannheim K, Shainker SA, Hecht JL. Hysterectomy for_placenta_accreta; methods for gross and microscopic pathology examination. Arch Gynecol Obstet. 2016;293:951-8.

22. Jauniaux E, Ayres-de-Campos D, Langhoff-Ross J, Fox KA, Collins SL, FIGO Placenta Accreta Diagnosis and Management Expert Consensus Panel. FIGO classification for the clinical diagnosis of placenta accreta spectrum disorders. Int J Gynecol Obstet 2019;146:20-4.

23. Collins SL, Alemdar B, van Beekhuizen HJ, Bertholdt C, Braun T, Calda P, et al; International Society for Abnormally Invasive Placenta (IS-AIP). Evidencebased guidelines for the management of abnormally invasive placenta: recommendations from the International Society for Abnormally Invasive Placenta. Am J Obstet Gynecol. 2019;220:511-26.

24. Collins SL, Ashcroft A, Braun T, Calda P, Langhoff-Ross J, Morel O et al., Proposed for standardized ultrasound descriptions of abnormally invasive placenta (AIP). Ultrasound Obstet Gynecol. 2016;47:271-275. 
25. Finberg HJ, Williams JW. Placenta accreta: prospective sonographic diagnosis in patients with placenta previa and prior cesarean section. $\mathrm{J}$ Ultrasound Med. 1992;11:333-43.

26. Collins SL, Stevenson GN, Al-Khan A, Illsley NP, Impey L, Pappas L, et al. Three-Dimensional Power Doppler Ultrasonography for Diagnosing Abnormally Invasive Placenta and Quantifying the Risk. Obstet Gynecol. $2015 ; 126: 645-53$

27. Cali G, Forlani F, Lees C, et al. Prenatal ultrasound staging system for placenta accreta spectrum disorders. Ultrasound Obstet Gynecol. 2019;53:752-60.

28. Chantraine F, Blacher S, Berndt S, Palacios-Jaraquemada J, Sarioglu N, Nisolle $\mathrm{M}$, et al. Abnormal vascular architecture at the placental-maternal interface in placenta increta. Am J Obstet Gynecol. 2012;207:188.e1-9.

29. Breen JL, Neubecker R, Gregori CA, Franklin JE Jr. Placenta accreta, increta, and percreta. A survey of 40 cases. Obstet Gynecol. 1977;49:43-7.

30. Parra-Herran C, Djordjevic B. Histopathology of placenta creta: chorionic villi intrusion into myometrial vascular spaces and extravillous trophoblast proliferation are frequent and specific findings with implications on diagnosis and pathogenesis. Int J Gynecol Pathol. 2016;35:497-508.

31. Schaaps JP, Tsatsaris V, Goffin F, et al. Shunting the intervillous space: new concepts in human uteroplacental vascularization. Am J Obstet Gynecol. 2005;192:323-32.

32.Zosmer N, Jauniaux E, Bunce C, Panaiotova J, Shaikh H, Nicholaides KH. Interobserver agreement on standardized ultrasound and histopathologic 
signs for the prenatal diagnosis of placenta accreta spectrum disorders. Int $\mathrm{J}$ Gynaecol Obstet. 2018;140:326-331. 
Table 1. Comparison of maternal demographics and PAS pathology technical characteristics for the study $(n=24)$ and control $(n=24)$ groups.

Variable

Study

Controls

$\mathbf{P}$ Group

\begin{tabular}{|l|l|l|l|}
\hline Maternal age (years) & $32.5(29.5 ; 35.0)$ & $35.5(32.5 ; 38.5)$ & $\mathbf{0 . 0 0 4}$ \\
\hline Gravidity & $5.0(3.5 ; 6.0)$ & $4.0(3.0 ; 6.0)$ & 0.430 \\
\hline Parity & $3.0(2.0 ; 4.5)$ & $3.0(2.0 ; 3.0)$ & 0.08 \\
\hline No of prior Caesarean sections & $3.0(2.0 ; 4.0)$ & $2.0(1.0 ; 3.0)$ & $\mathbf{0 . 0 0 5}$ \\
\hline Gestational age at delivery & $36.3(36.0-37.1)$ & $35.8(33.7-36.8)$ & 0.189 \\
\hline $\begin{array}{l}\text { Histopathology reporting time } \\
\text { (days) }\end{array}$ & $7.0(7.0 ; 8.0)$ & $15.5(12.5 ; 22.5)$ & $<0.001$ \\
\hline $\begin{array}{l}\text { No of slides showing invasive } \\
\text { villi }\end{array}$ & $2.0(2.0 ; 3.0)$ & $1.0(0.5 ; 2.0)$ & $\mathbf{0 . 0 0 2}$ \\
\hline $\begin{array}{l}\text { No of samples taken per } \\
\text { hysterectomy specimen }\end{array}$ & $4.0(3.0 ; 5.0)$ & $14.0(12.0 ; 15.0)$ & $<\mathbf{0 . 0 0 1}$ \\
\hline $\begin{array}{l}\text { No of samples taken/ slides } \\
\text { showing invasive villi }\end{array}$ & $2.0(1.5 ; 2.0)$ & $9.0(7.0 ; 15.0)$ & $<\mathbf{0 . 0 0 1}$ \\
\hline $\begin{array}{l}\text { PAS grading Adherent }(\mathrm{n}) \\
\text { Invasive }(\mathrm{n})\end{array}$ & $\begin{array}{l}2(8.3 \%) \\
22(91.7 \%)\end{array}$ & $\begin{array}{l}7(29.2 \%) \\
17(70.8 \%)\end{array}$ & $0.139^{*}$ \\
\hline
\end{tabular}

PAS: Placenta accreta spectrum. ${ }^{*}$ Chi-square with Yates correction.

Numerical data are presented as median (interquartile range) and categorical data as $\mathrm{n}(\%)$. 
Table 2. Ultrasound and peri-operative macroscopic characteristics of the study group $(n=24)$.

\section{Characteristics}

Cases n (\%)

\begin{tabular}{|c|c|}
\hline Placental location & \\
$-\quad$ Low-lying & $1(4.2)$ \\
$-\quad$ Marginal previa & $1(4.2)$ \\
$-\quad$ Covering previa & $22(91.6)$ \\
\hline Myometrium thickness & \\
$-\quad$ - $1 \mathrm{~mm}$ (Thin) & $19(79.2)$ \\
$-1-2 \mathrm{~mm}$ & $3(12.5)$ \\
$-\quad 2-3 \mathrm{~mm}$ & $2(8.3)$ \\
\hline Lacunae score & $3(12.5)$ \\
$-\quad 1+(1-3)$ & $7(29.2)$ \\
$-\quad 2+(4-6)$ & $14(58.3)$ \\
$-\quad 3+(>6)$ & \\
\hline Intra-operative anterior wall dehiscence & $2(8.3)$ \\
$-\quad$ None & $10(41.7)$ \\
- Focal & $12(50.0)$ \\
$-\quad$ Large & $2(8.3)$ \\
\hline Immediate post-operative PAS grading \& area & $9(37.5)$ \\
$-\quad$ Adherent & $13(54.2)$ \\
- Focal invasive & \\
- Large invasive & \\
\hline
\end{tabular}


Table 3. Ultrasound and peri-operative macroscopic correlations for the invasive cases of the study group $(n=22)$.

\begin{tabular}{|c|c|c|c|c|c|}
\hline Ultrasound features & \multicolumn{3}{|c|}{ Anterior wall dehiscence } & \multicolumn{2}{|c|}{ Invasive area } \\
\hline $\begin{array}{l}\text { Focal } \\
n=9\end{array}$ & $\begin{array}{c}\text { Large } \\
n=11\end{array}$ & $\begin{array}{c}\text { Focal } \\
n=9\end{array}$ & $\begin{array}{c}\text { Large } \\
n=13\end{array}$ & & \\
\hline $\begin{array}{c}\text { Myometrium thickness } \\
-\quad<1 \mathrm{~mm} \text { (Thin) } \\
-\quad 1-2 \mathrm{~mm} \\
-\quad 2-3 \mathrm{~mm}\end{array}$ & $\begin{array}{l}0 \\
1 \\
1\end{array}$ & $\begin{array}{l}7 \\
1 \\
1\end{array}$ & $\begin{array}{r}10 \\
1 \\
0\end{array}$ & $\begin{array}{l}7 \\
0 \\
2\end{array}$ & $\begin{array}{r}10 \\
3 \\
0\end{array}$ \\
\hline $\begin{array}{r}\text { Lacunae score } \\
-\quad 1+(1-3) \\
-\quad 2+(4-6) \\
-\quad 3+(>6)\end{array}$ & $\begin{array}{l}1 \\
1 \\
0\end{array}$ & $\begin{array}{l}0 \\
3 \\
6\end{array}$ & $\begin{array}{l}0 \\
3 \\
8\end{array}$ & $\begin{array}{l}0 \\
4 \\
5\end{array}$ & $\begin{array}{l}1 \\
3 \\
9\end{array}$ \\
\hline $\begin{array}{c}\text { Feeder vessels } \\
-\quad \text { None } \\
-\quad \text { Yes }\end{array}$ & $\begin{array}{l}2 \\
0\end{array}$ & $\begin{array}{l}3 \\
6\end{array}$ & $\begin{array}{l}4 \\
7\end{array}$ & $\begin{array}{l}3 \\
6\end{array}$ & $\begin{array}{l}6 \\
7\end{array}$ \\
\hline
\end{tabular}




\section{FIGURE LEGENDS}

Figure 1. Intra-operative views uterine wall dehiscence (D) in cases of placenta previa increta. Note tangled bed of vessels and multiple vessels running craniocaudally and laterally in the anterior perimetrium in both cases. Left) anterior and lateral focal areas of dehiscence; Right) large area of dehiscence (uterine window) occupying most of the lower uterine segment.

Figure 2. Intra-operative views showing dense tangled bed of vessels and multiple vessels running cranio-caudally and laterally in the anterior perimetrium in cases of placenta previa increta. Left) There is no evidence of uterine dehiscence in this case; Right) Note the presence of small area of anterior wall uterine dehiscence (D).

Figure 3. Immediate post-operative views of hysterectomy specimen before and after dissection in a case of placenta previa increta. Left) Note a large area of anterior wall uterine dehiscence (D) underneath which part of the placenta is inserted. The hypervascularity of the serosa is no longer visible; Middle) View of a longitudinal uterine slice showing the placental insertion $(\mathrm{P})$. Note the presence of dilated vessels (arrows) protruding from the uterine $(U)$ wall towards the uterine cavity, above the placental insertion. The placental tissue between the 2 arrows corresponds to the placental portion visible under the serosa of the uterine dehiscence; Right) View of the same slice after digital dissection. The portion of the placenta that could not be digitally separated (*) showed villous tissue invading the myometrium on microscopic examination.

Figure 4. Immediate post-operative views of hysterectomy specimen before and after dissection in a case of placenta previa increta. Left) Note a large area of anterior wall uterine dehiscence (D); Right) View after dissection showing a large portion of the placenta deeply embedded within the uterine wall $(P)$ which could not be digitally separated during dissection.

Figure 5. Immediate post-operative view of hysterectomy specimen after dissection in a case of abnormally adherent placenta previa. The placenta could be completely digitally separated from the uterine wall.

Figure 6. Immediate post-operative views of hysterectomy specimens after dissection in cases of increta placentation showing dilated vessels (arrows) protruding from the uterine $(\mathrm{U})$ wall towards the uterine cavity, away from the placental $(\mathrm{P})$ insertion. 